\title{
Self-expandable metal stent placement in a child for treatment of achalasia after failed Heller myotomy
}

\section{(ㄷ)(구)(우}

Roberto Gugig², Guillermo Muñoz Jurado ${ }^{1}$, Clifton Huang ${ }^{2}$, Roberto Oleas ${ }^{1}$, Carlos Robles-Medranda ${ }^{1}$

\author{
Authors \\ Institutions \\ 1 Instituto Ecuatoriano de Enfermedades Digestivas, \\ University Hospital OMNI, Guayaquil, Ecuador \\ 2 University of California San Francisco and Valley \\ Children's Healthcare, San Francisco, California, United \\ States \\ submitted 26.2.2017 \\ accepted after revision 24.7.2017 \\ Bibliography \\ DOI https://doi.org/10.1055/s-0043-118745 | \\ Endoscopy International Open 2018; 06: E64-E66 \\ (c) Georg Thieme Verlag KG Stuttgart · New York \\ ISSN 2364-3722 \\ Corresponding author \\ Carlos Robles-Medranda, IECED, Omni Hospital, Av. Abel R \\ Castillo y Av. Juan Tanca Marengo s/n, Ciudad del Sol, Office \\ Mezanine 3, 090505, Guayaquil, Guayas, Ecuador \\ Fax: +59342109180 \\ carlosoakm@yahoo.es
}

\section{ABSTRACT}

Background and study aims Childhood achalasia treatment remains inconclusive. What is next after myotomy failure? Repeated pneumatic-dilation put patients at greater risk of perforation with possible symptom recurrence. We report on a 12-year-old patient with a 1-year history of achalasia whom underwent Heller myotomy with fundoplication and recurred with symptoms 1 week after surgery. Pneumatic dilatation was considered but not done because of the risk of esophageal perforation. The decision was made to place a fully covered self-expanding metallic stent (FC-SEMS) for 3 months, which resolved the stenosis as confirmed by esophagram. The patient has remained asymptomatic since the procedure was performed 2 years ago. FCSEMS is an alternative for treatment of refractory achalasia in children who do not respond to conventional treatment.

\section{Introduction}

Achalasia is a relatively uncommon disorder of the esophagus affecting the lower esophageal sphincter (LES), resulting in dysphagia [1]. Treatment of Achalasia is directed toward decreasing the resting pressure at the LES by: pneumatic dilation (PD) [1], per oral endoscopy myotomy (POEM) [2], or laparoscopic Heller myotomy (LHM) or biochemical reduction in the LES pressure (Botulinum toxin, oral nitrates, etc.). PD is the most effective non-surgical treatment. The short- to medium-term effects are good, nevertheless, up to $66 \%$ of these patients may need reintervention 5 to 10 years later. Moreover, the risk of esophageal perforation (EP) ranges from $1 \%$ to $5 \%$ [3]. The question is what can be done if symptoms recur after myotomy and the answer remains unclear. In the pediatric population, after myotomy, performing PD greatly increases the risk of EP. Self-expanding metallic stents (SEMS) are the most common form of palliative treatment for malignant esophageal dysphagia and/or strictures. Today fully-covered SEMS (FC-SEMS) are formally indicated for benign esophageal conditions. Minimal published reports exist about use of SEMS in achalasia in an adults [4].

We report on a 12-year-old boy who presented with recurrent achalasia symptoms after LHM who underwent placement of FC-SEMS.

\section{Case report}

A 12-year-old male initially presented with a 12-month history of progressive dysphagia and emesis. On initial diagnosis, upper gastrointestinal series (UGIs) showed a bird's beak with a dilated esophagus proximal to the stenosis. Esophageal manometry and Chagas serology confirmed the diagnosis of achalasia type I (Chicago Classification) not secondary to Chagas. The patient underwent a 4-cm LHM and fundoplication. Concern for dysphagia continued after surgery and persisting stenosis was evidenced by 2 UGls performed post-surgery and 1 week after the procedure. Four months after myotomy the patient was first seen at our institution. His Eckardt score was 9 (daily dysphagia, regurgitation with each meal, occasional retrosternal 


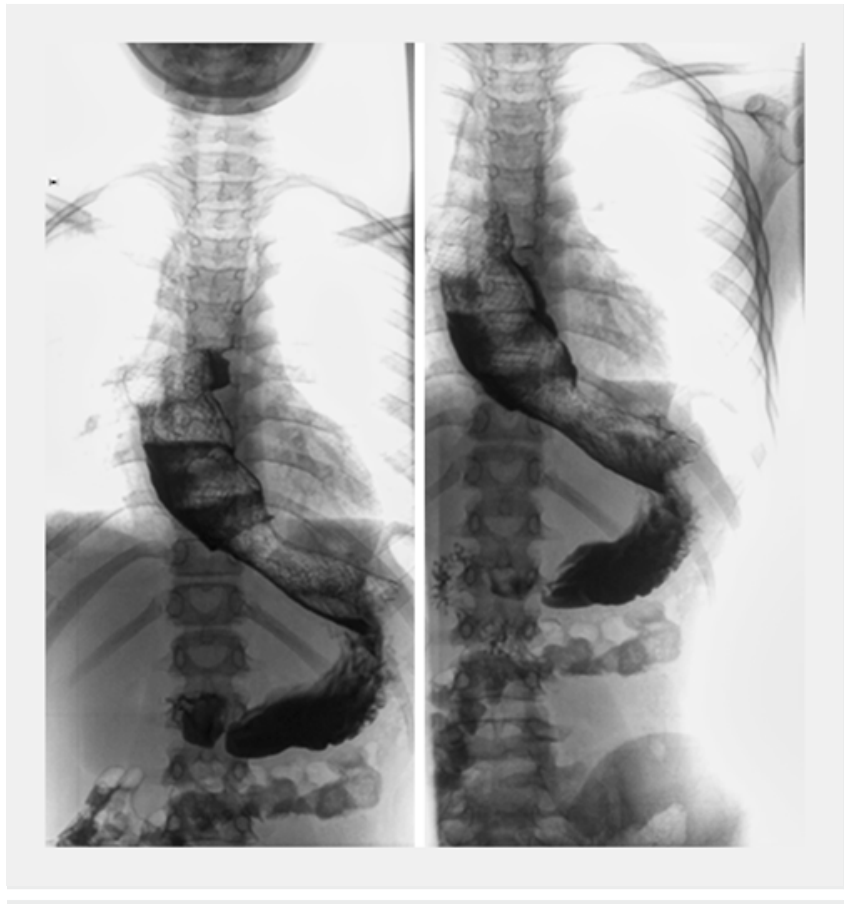

- Fig. 1 Stent placement.

pain and $>10 \mathrm{~kg}$ of weight loss). We performed esophageal manometry, which confirmed the diagnosis of achalasia type I. Due to a previous LHM, posterior POEM was considered as an alternative, however, the procedure was not performed in the country by any institution. Moreover, performing PD is associated with a concern about EP and a decision was made to place a stent [Niti-S Esophageal Covered Stent (Double-type) Taewoong Medical] $30 \mathrm{~mm}$ in diameter and $60 \mathrm{~mm}$ long (after approval by the ethics committee of our institution and signing of informed consent by the boy's parents). The patient reported retrosternal discomfort during the first 48 hours, which was treated with oral paracetamol. There were no major adverse events (AEs) post-procedure ( $\mathbf{F i g} \mathbf{1} \mathbf{1})$.

One month after stent placement, UGls showed normal gastrointestinal transit. The stent was retrieved 3 months after with esophagogastroduodenoscopy and UGIs showed no distal esophageal stenosis at 6 months ( $\triangleright$ Fig. 2). After a follow up of 2 years, the patient reported no dysphagia (Eckardt score $<3$ ) and had weight gain and growth appropriate for his age.

\section{Discussion}

In patients with achalasia who are low-risk surgical candidates, either a graded PD or LHM with a fundoplication should be performed [1]. However, the efficacy of both treatments decreases overtime [1,3].

Multiple studies suggest that in older children, PD is effective and safe as treatment for achalasia [5,6]. However, multiple PDs are required. Hamza et al [6], reported a $90 \%$ success rate in children treated with multiple PDs. Adverse events related to PD are: prolonged epigastric pain, esophageal perforation, and aspiration pneumonia [5]. In children, surgery is the

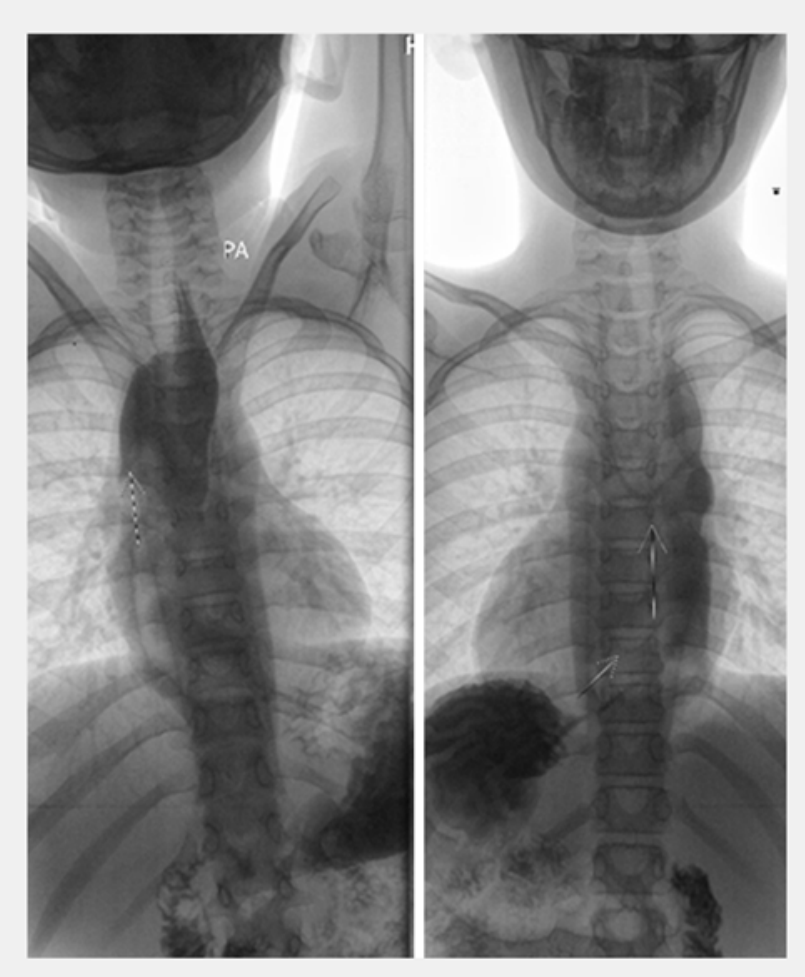

Fig. 2 Upper gastrointestinal series made 6 months after stent placement and showing no stenosis.

treatment of choice (open or LHM). However, 7.3\% developed major AEs of esophageal perforation and dysphagia (11.7\%) [7].

Uncovered SEMS are contraindicated for benign pathology because of risk of stent-induced tissue hyperplasia, which will lead to failure of stent removal. However, that risk is eliminated by using a FC-SEMS as long as it is placed for not more than 3 months to avoid risk of stent migration [3].

Cheng et al demonstrated the adequate outcome and safety of large SEMS placed temporarily across the cardia in adults. Recently, the same authors confirmed long-term results obtained with temporary stents and compared the efficacy of different diameters, demonstrating that stents with a $30-\mathrm{mm}$ caliber are more effective after 10-year follow-up [8].

Recently, Cai et al. showed that SEMS was superior to intrasphincter injection of botulinum toxin for global symptoms, dysphagia scores and basal lower esophageal sphincter pressure and obtained better remission rates after 12 and 36 months of follow-up [9].

It is unknown why a temporary stent should work better than a PD. We speculate that prolonged dilation obtained with a large stent is truly effective because it permanently disrupts the muscular fibers of the LES. The procedure seems to be safe and effective. When compared to PD, the primary benefit is avoidance of perforations. Moreover, fewer procedures are required with lower rates of symptom recurrence $[10,11]$. 


\section{Conclusion}

Treatment options for achalasia remain palliative. In this setting, where our patient had no initial response to LHM plus the risk of EP with subsequent PD, a FC-SEMS was considered a safe and effective alternative. At 24 months' post-treatment, our patient remains asymptomatic with no dysphagia as evidenced by normal UGIs.

Several technical issues including stent type, diameter, length, removal time and prevention of complications are still open for discussion in children. Large, multicenter, randomized trials are warranted to elucidate the role of FC-SEMS in the treatment of children with achalasia refractory to esophageal myotomy.

Competing interests

None

References

[1] Vaezi MF, Pandolfino JE, Vela MF. ACG clinical guideline: diagnosis and management of achalasia. Am J Gastroenterol 2013; 108: 1238

[2] Chen WF, Li QL, Zhou PH et al. Long-term outcomes of peroral endoscopic myotomy for achalasia in pediatric patients: a prospective, single-center study. Gastrointest Endosc 2015; 81: 91 - 100
[3] Chuah S-K, Chiu C-H, Tai W-C et al. Current status in the treatment options for esophageal achalasia. World J Gastroenterol 2013; 19: $5421-5429$

[4] Zhao JG, Li YD, Cheng YS et al. Long-term safety and outcome of a temporary self-expanding metallic stent for achalasia: A prospective study with a 13-year single-center experience. Eur Radiol 2009; 19: $1973-1980$

[5] Babu R, Grier D, Cusick E et al. Pneumatic dilatation for childhood achalasia. Pediatr Surg Int 2001; 17: 505-507

[6] Hamza AF, Awad HA, Hussein O. Cardiac achalasia in children. Dilatation or surgery? Eur J Pediatr Surg 1999; 9: 299-302

[7] Franklin A, Petrosyan M, Kane TD. Childhood achalasia: A comprehensive review of disease, diagnosis and therapeutic management. World J Gastrointest Endosc 2014; 6: 105-111

[8] Cheng YS, Ma F, Li YD et al. Temporary self-expanding metallic stents for achalasia: a prospective study with a long-term follow-up. World J Gastroenterol 2010; 16: 5111 - 5117

[9] Cai XB, Dai YM, Wan X] et al. Comparison between botulinum injection and removable covered self-expanding metal stents for the treatment of achalasia. Dig Dis Sci 2013; 58: 1960 - 1966

[10] Coppola F, Gaia S, Rolle E et al. Temporary Endoscopic Metallic Stent for Idiopathic Esophageal Achalasia. Surg Innov 2014; 21: 11- 14

[11] Li YD, Tang GY, Cheng YS et al. 13-Year follow-up of a prospective comparison of the long-term clinical efficacy of temporary self-expanding metallic stents and pneumatic dilatation for the treatment of achalasia in 120 patients. Am J Roentgenol 2010; 195: 1429-1437 